\title{
Preference-Based Group Scheduling
}

\author{
Jiang Hu and Mike Brzozowski \\ Stanford University \\ \{huj, zozo\}@Stanford.edu
}

\begin{abstract}
Traditional group scheduling applications often treat users' availability as binary: free or busy. This is an unrealistic representation because not all times are equally free or busy. The inflexibility makes it difficult to find times with which everyone is truly satisfied. We present an online group scheduling approach by which users indicate four-tier preferences for meeting times on a calendar, which dynamically adjusts to provide instant feedback and suggests optimal meeting times for all participants. Our prototype is geared toward college students and the scheduling is done through a democratic and open negotiation process where everyone's preference is heard. Students who evaluated the prototype thought that the scheduling process was more efficient than the widely-used e-mail scheduling among college students, which is largely based on binary availability.
\end{abstract}

\section{Introduction}

Even though many group scheduling applications are commercially available [1], they are still considered "the least useful groupware application" [2]. Group scheduling has come to be viewed, as Palen [3] describes it, as "less an 'optimizing' task and more often a 'satisficing' task." A primary limitation of traditional group scheduling systems is that they treat users' time as binary: free or busy. The inflexibility of such treatments tends to make group scheduling a lengthy and frustrating process. In reality, not all free times are created equal, nor are all busy times absolutely immutable. This is especially true for college students.

Unlike business users, college students have very erratic schedules and often find themselves "at work" $24 \times 7$. During academic quarters/semesters, students have to meet in groups for class projects, assignments, social activities, and so on. However, when the meeting involves multiple students who are not often in the same place to discuss availability, scheduling becomes difficult.

Current groupware scheduling systems are expensive and typically designed for corporate users. Our own experience and interviews with fellow students, however, suggest that college students also desire a way to easily schedule meetings with a minimum of effort, and without constant maintenance of an additional calendar. In fact, many universities nowadays provide students with online registration services that generate weekly schedules on a quarter or semester basis. Group scheduling systems for students can readily tap into existing online registration systems and directly address many of Grudin's concerns over groupware [2]. 


\section{Preference-Based Group Scheduling}

Beard et al.'s Visual Scheduler [4] introduced a "priority-based" scheduling system, allowing users to mark any given busy time slots with five levels of gray shading to indicate "low" to "highest" priority. However, no attempt was made to distinguish between better or worse free times. In general, Visual Scheduler and similar systems operate within the relatively controlled domain of an office environment.

Here we introduce a scheduling system based not on users' absolute availability as a traditional "free/busy" binary but rather on users' preferences, extending the Visual Scheduler model by allowing users to indicate their better and worse free times as well as busy times. The system is designed so that it is to each user's advantage to respond quickly to a meeting request.

\subsection{Contextual Inquiry}

In order to have a clear picture of students' group scheduling behaviors, we observed and interviewed some students from an introductory HCI class at Stanford University. We found that students in general use e-mail to schedule meetings when it is impractical do so face-to-face. To simulate the common scenario where a professor groups students in project teams, we e-mailed five groups of four randomly selected student volunteers, asking each group to find a good time for an ostensibly "focus group" meeting on interface design.

To our surprise, only three groups finished the scheduling task and they all used email as the primary means of communication. Inferring from our correspondence with the failing groups, the frustration associated with the e-mail scheduling process appeared to be a significant barrier to completing the task.

Most importantly, we observed that asynchronous group scheduling typically takes one of two routes: 1) Negotiation: One student initiates an e-mail thread by enumerating several potential meeting times he/she is free to seek other students' opinions on those times. As others respond, a consensus is reached by process of elimination; 2) Aggregation: One student from the group sends an e-mail asking for everyone's availabilities. After getting every member's schedule, the student manually combines them and selects a meeting time for the group. In either case, the inherent problem with binary availability was frequently pronounced in the form of multiple rounds of negotiation and involuntary acceptance of sub-optimal times.

\subsection{The Prototype}

Our prototype blends the two strategies by aggregating user calendars to automatically select optimal meeting times and then letting users negotiate through providing additional information about their time constraints and preferences. The prototype takes the form of an Excel workbook. Each worksheet of the workbook contains a grid of a user's schedule for the following week. Every cell in this grid represents a 30-minute block and can be marked/colored with one of the following labels (borrowed from Evite [5]):

- Works Great: "This is one of the best times for me."

- Is $O K$ : "I could come, but there are other times that work better." 
- Rather Not: "I'd rather not do it then but I could come if I had to."

- Can't Make It: "There is no way I can meet then."

Depending on the color assigned, each cell is assigned a numeric value from 0 (Can't Make It) to 3 (Works Great). Cells without a color are treated as "Is OK," receiving the value of 2 , because people are generally apathetic about times that are acceptable to them. Also, this provides extra incentive to people to correct the model if it is actually a bad time.

On the worksheet that aggregates all group members' schedules, each cell's score is computed as the average of the value each user labeled it, and the optimal time periods for meeting are those that span the maximal set of scores, as those are the times that maximize overall satisfaction within the group. To avoid confusion, we restrict the best times selected so that they do not overlap.

During the set-up stage, users are encouraged to label their firm time commitments with one of the latter two categories so the coloring closely corresponds to their quarterly schedules. When a user receives a workbook during the negotiation process, the application has suggested five times that it thinks are optimal. The user can respond by coloring cells in and near the proposed time. Upon making any change, the system automatically re-computes the best options, so the user can ensure that the proposed times are acceptable before forwarding the workbook on to the next user.

\section{Evaluation and Results}

Twenty students from an introductory communication class participated in the user study for course credit. Upon signing up, the 12 male students and 8 female students were randomly assigned to five groups of four students.

There are three major steps. First, each group was given instructions similar to those used in our initial contextual inquiry except that these five new groups had to identify two possible times for a focus group meeting. Second, during the meeting at a scheduled time, participants used our prototype to schedule another hour-long meeting for the coming week. Their first subtask was to label firm time commitments based on their quarterly schedules as if this was done at the start of the quarter/semester. The second subtask was to check the top five meeting times recommended by the prototype, and to modify their schedules for the coming week so as the prototype could adjust its recommendations based on accurate schedules and time preferences. The order in which they took turns to modify their calendars was identical to the order in which they first started/responded to their scheduling e-mail threads. Lastly, the participants filled out an online questionnaire to compare the second subtask with e-mail scheduling.

In the first step, four of the five groups were able to identify only one possible meeting time via e-mail; one of the four groups did not evaluate the prototype due to the absence of one group member. E-mail records indicate that all four groups went through negotiation instead of aggregation. On average, each student had to read about seven e-mails before a mutually acceptable meeting time was selected.

With the help of the prototype's aggregation, however, three of the four groups finished their scheduling task with five possible meeting times after only one round of negotiation. This is far more efficient than e-mail scheduling. As a result, participants 
compared the prototype favorably with conventional scheduling using e-mail: they thought the prototype was "easy to use" $\left[M_{\text {easy }}=8.31, \mathrm{SD}=1.71\right.$, on a 10-point Likert scale], and "user-friendly" $\left[M_{\text {friendly }}=8.13, \mathrm{SD}=1.25\right]$. They also indicated high likelihood to try out an application based on our prototype and to recommend it to friends and classmates.

\section{Conclusion}

Our preference-based scheduling system shortens the negotiation process during group scheduling and recommends multiple, ranked, meeting times. We have identified a new segment of users underserved by current scheduling solutions, and applied the insights gained through contextual inquiry to construct the prototype. As our evaluation indicates, greater satisfaction with the meeting times should be expected, potentially contributing to harmonious and productive collaborations among meeting attendees.

We evaluated our prototype against scheduling through e-mail, but the advantages the prototype holds may well extend to other scheduling systems based on binary availability. The preference-based principle can be applied to other contexts than college campus. If synchronized with actively used personal or group calendars such as Microsoft Outlook or a PDA, such a system could become even more powerful.

The model we present also provides a framework for automated optimal scheduling, potentially based on incomplete information. We demonstrated that users are comfortable negotiating with this model, providing a platform on which to test probabilistic and empirical techniques for hypothesizing about preferences.

\section{References}

1. Palen, L, Grudin, J.: Discretionary Adoption of Group Support Software: Lessons from Calendar Applications. Implementing Collaboration Technologies in Industry. SpringerVerlag, Berlin Heidelberg New York (2003) 159-179

2. Grudin, J.: Groupware and Social Dynamics: Eight Challenges for Developers. Comm. of the ACM January (1994) 93-105

3. Horvitz, E., Koch, P., Kadi, C. M. Jacobs, A.: Coordinate: Probabilistic Forecasting of Presence and Availability. Proc. Of the $18^{\text {th }}$ Conference on Uncertainty and Artificial Intelligence. Morgan Kaufman (2002) 224-233

4. Beard, D., Palaniappan, M., Humm, A, Banks, D., Nair, A., Shan, Y.: A Visual Calendar for Scheduling Group Meetings. Proc. of the 1990 ACM Conference on CSCW. ACM Press (1990) 279-290

5. Evite. http://www.evite.com

6. Tullio, J., Goecks, J., Mynatt, E.D., Nguyen, D. H.: Augmenting Shared Personal Calendars. Proc. of the $15^{\text {th }}$ Annual ACM Symposium on UIST. ACM Press (2002) 11-20 